\title{
Lumen
}

Selected Proceedings from the Canadian Society for Eighteenth-Century Studies

\section{Aspects bibliographiques du paratexte chez Mme de Graffigny $-2$}

\section{David Smith}

Volume 38, 2019

URI : https://id.erudit.org/iderudit/1059275ar

DOI : https://doi.org/10.7202/1059275ar

Aller au sommaire du numéro

Éditeur(s)

Canadian Society for Eighteenth-Century Studies / Société canadienne d'étude du dix-huitième siècle

ISSN

1209-3696 (imprimé)

1927-8284 (numérique)

Découvrir la revue

Citer cet article

Smith, D. (2019). Aspects bibliographiques du paratexte chez Mme de Graffigny - 2. Lumen, 38, 105-115. https://doi.org/10.7202/1059275ar d'utilisation que vous pouvez consulter en ligne. 


\title{
Aspects bibliographiques du paratexte chez Mme de Graffigny - 2
}

\author{
David Smith \\ Université de Toronto
}

Des trois catégories de paratexte établies par Gérard Genette dans son ouvrage pionnier intitulé Seuils ${ }^{1}$, le paratexte bibliographique, ensemble hétéroclite d'aspects, n'est sans doute pas le moins utile aux chercheurs, même s'ils ne sont pas bibliographes. J'ai choisi d'examiner de manière systématique les aspects bibliographiques du paratexte de Mme de Graffigny, comme il est naturel pour quelqu'un qui a consacré plus de trente ans à préparer une bibliographie de ses œuvres ${ }^{2}$. Le premier volet de mes remarques a fait l'objet d'une communication que j'ai faite il y a deux ans à Vancouver et qui a été publiée dans Lumen³. J'y ai traité de certains aspects tels que reliure, ex-libris, faux-titre, page de titre, dédicace, format, signatures et réclames. Le présent article constitue le second volet de mes remarques. J'y étudie le frontispice, les préfaces, la distribution des rôles, les ornements, les estampilles, le papier, l'approbation, le privilège, l'errata, les éditions révisées et les traductions.

Le frontispice. Aucun portrait de l'auteure ne figure dans les éditions publiées de son vivant. On en trouve un pour la première fois en 1777 (P.55). Le portrait, comme les autres planches, était parfois offert

1. Paratexte littéraire, illustrations, bibliographie matérielle (Gérard Genette, Seuils, Paris, Seuil, 1987, p. 7 et 20. Les illustrations sont le domaine d'élection de Christina Ionescu).

2. David Smith, Bibliographie des oeuvres de Mme de Graffigny, Ferney-Voltaire, Centre international d'étude du XVIII ${ }^{\mathrm{e}}$ siècle, 2016. Nous employons dans le texte les sigles de cet ouvrage pour les différentes éditions qui y figurent. Une liste détaillée des éditions citées figure à la fin du présent article.

3. David Smith, "Aspects bibliographiques du paratexte chez Mme de Graffigny », Lumen, 36, 2017, p. 63-73. 
en option, de sorte qu'on le trouve souvent relié dans une édition à laquelle il n'était pas destiné. Bien sûr, le frontispice n'est pas toujours un portrait; il peut être aussi une scène du texte (C.18). En général on le trouve en face de la page de titre, mais dans la traduction hollandaise de Cénie (C.18), le frontispice tourne le dos au titre, ce qui doit être une pratique des relieurs néerlandais de l'époque.

La préface. La Péruvienne (P.1) est l'unique ouvrage de Graffigny à comporter une préface. Elle est parfaitement typique de celles de l'époque. Comme l'a bien montré Vivienne Mylne dans Techniques of Illusion, un des procédés favoris des romanciers français du XVIII siècle pour emporter l'adhésion des lecteurs était de nier la paternité de leur ouvrage, qu'il s'agisse d'un roman en forme de mémoires ou de lettres ${ }^{4}$. Graffigny se pose donc en «éditeur» de lettres réelles écrites par Zilia et à lui communiquées par Déterville qui a obtenu la permission de Zilia de les publier. Notons en passant que les lettres d'une femme écrites en fait par une femme doivent donc passer par un personnage masculin ainsi qu'un éditeur masculin; du point de vue féministe, c'est assez décevant. L'auteure atteste la vérité de ces lettres: le roman n’est peut-être pas vraisemblable, mais il est véridique. Le caractère fictif de l'héroïne reste quand même ambigu : «L'on ne devrait pas craindre de voir passer pour une fiction des lettres originales ${ }^{5}$ ». C'est-à-dire, les lettres semblent fictives, mais elles ne le sont pas, mais le lecteur averti sait parfaitement qu'elles le sont! Pour corroborer la véracité de ces lettres d'une étrangère, Graffigny explique qu'elles contiennent des «fautes de grammaire» et des «négligences du style ${ }^{6}$, ce qui lui permet en même temps de s'excuser modestement de ses propres défauts. Le soi-disant éditeur indique aussi qu'il a supprimé « un grand nombre de termes et de comparaisons orientales ${ }^{7} »$. Orientales?! Mais le Pérou est situé plutôt à l'occident; le terme «orientales » ne figurera plus dans le texte révisé de 1752. Selon l'éditeur, il a également rendu certaines notions métaphysiques plus intelligibles au public français. «C'est la seule part que l'on ait à ce singulier ouvrage ${ }^{8} »$.

4. Voir Vivienne Mylne, The Eighteenth-Century French Novel. Techniques of Illusion, $2^{\mathrm{e}}$ éd., Cambridge U. P., 1981, chap. II, III et VIII.

5. Lire P.1, p. iv.

6. P.1, p. vi.

7. Ibid.

8. P.1, p. vii. 
Un autre but de cette préface est de préparer le lecteur à sa lecture du roman. L'auteure veut profiter de l'exotisme du roman, mais démontrer en même temps que les Péruviens sont des êtres humains comme nous; par exemple, elle parle de «la sagacité de leur esprit ${ }^{9} »$. Elle doit expliquer également que Zilia a employé des quipos (ou des nœuds de laine) pour écrire les premières lettres, mais qu'elle les a traduites lorsqu'elle a appris le français. (Il est fâcheux que la première lettre de Zilia, écrite en quipos, ait été dénouée par Aza, qui a ensuite utilisé les mêmes quipos pour écrire sa réponse. Mais n’en parlons pas - Zilia savait sans doute sa lettre par cœur).

Cette préface est atypique dans la mesure où Graffigny, volontiers moralisatrice d'ordinaire, ne proteste pas ici de la valeur morale de son roman. On s'attend enfin à ce qu'elle explique en quoi son roman ressemble à d'autres romans tels que les Lettres persanes. Elle cite sans contexte la question "Comment peut-on être Persan? », et mentionne un «crayon des mœurs indiennes dans un poème dramatique ", ce qui est, selon une note ajoutée à l'édition de 1752, une allusion à Alzire de Voltaire. Cette préface, révisée pour l'édition de 1752, est généralement conservée dans les traductions.

Cénie n'a pas de préface, mais les traductions anglaises en ont ajouté de leur cru. Celle de Cenia (C.8) nous informe que la traduction a été refusée tant à Covent Garden qu'à Drury Lane. Le prologue d'Eugenia (C.9), écrit et prononcé par David Garrick, explique que la pièce n'est pas une traduction mais une «imitation». Un épilogue, écrit par Colley Cibber et lu par la comédienne Mrs Pritchard, commente ainsi le texte original: «Such plays in French perhaps may cut a figure, / But to our critics here they're mere soup meagre». La fille d'Aristide n'a pas non plus de préface. Elle aurait pourtant gagné à être précédée d'un prologue explicatif prononcé par un comédien avant la représentation, comme le suggère Devaux dans une de ses lettres à l'auteure: «Il me semble [...] qu’on l'a jugée trop sévèrement. Le public n’a pas voulu se prêter à bien des choses sur lesquelles il aurait fallu le prévenir ${ }^{10} »$.

9. P.1, p. iii.

10. Françoise de Graffigny, Correspondance, éd. Alan Dainard et English Showalter, Oxford, Voltaire Foundation, 1985-2016, vol. 15, lettre 2430, 18 mai $175^{8}$. 


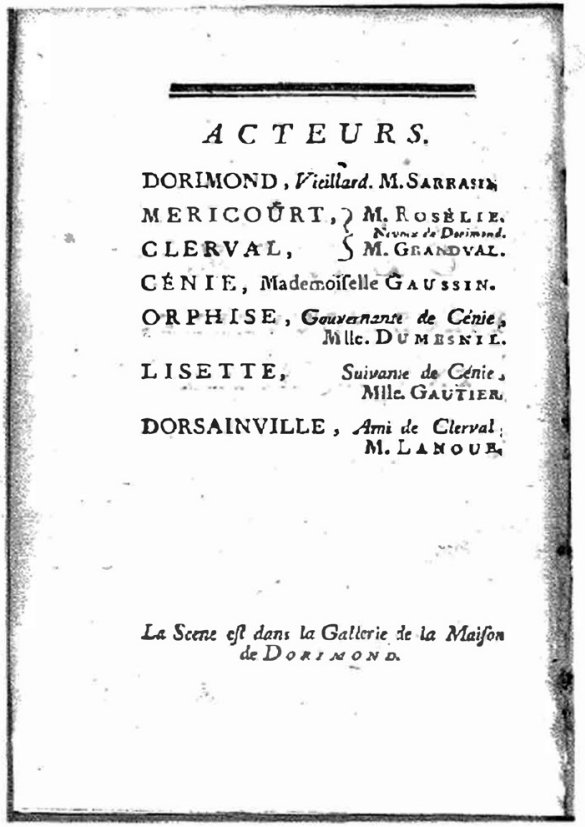

Planche 1
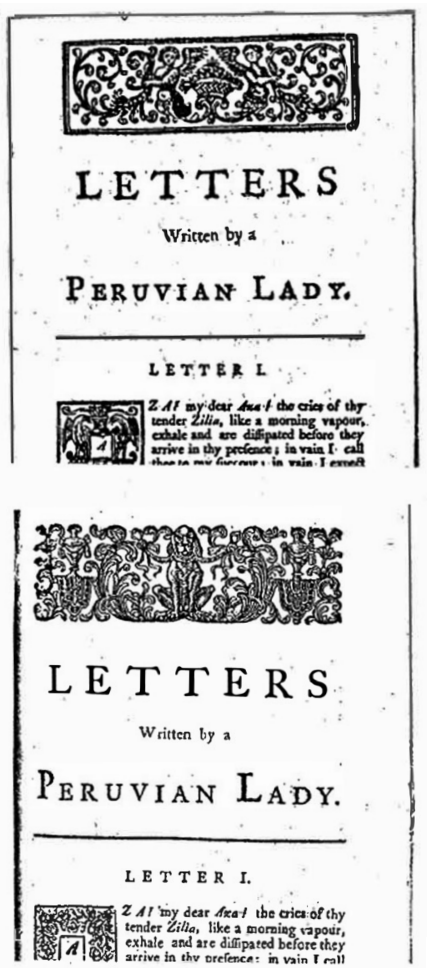

Planche 3

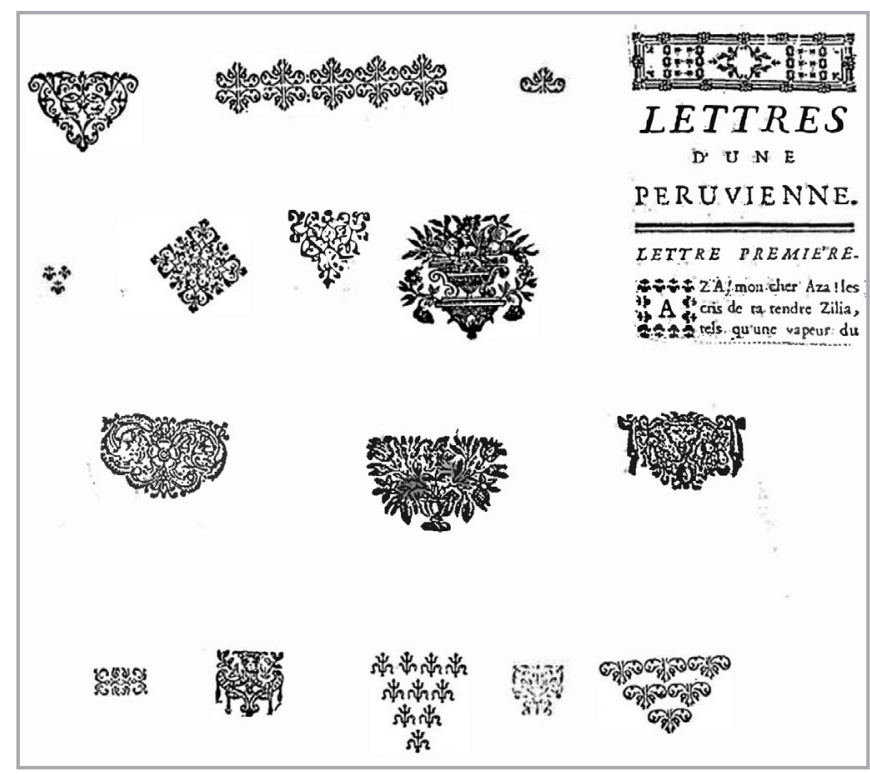

Planche 2 
Dramatis personce. En général, on trouvait sous cette rubrique non seulement les noms des personnages mais aussi ceux des acteurs, ce qui permettait aux lecteurs qui connaissaient les acteurs en question de les imaginer dans leurs rôles. Les noms des acteurs figurent dans Cénie (voir planche 1), mais non dans La fille d'Aristide, sans doute parce que la pièce a été un four. On trouve des éditions destinées à des représentations privées, parfois avec les noms des hauts personnages qui ont joué les rôles (voir, par exemple, A.3). Dans d'autres éditions, les noms des acteurs ont été ajoutés à la main, parfois avec la date de la représentation.

Les ornements. Les bibliographes utilisent les ornements pour identifier le libraire qui a publié une édition. Par exemple, les ornements fort reconnaissables de deux des premières éditions de la Péruvienne permettent de les attribuer à Aimé Delaroche de Lyon et à Everard Kints de Liège (P.5, P.g et planche 2). Les ornements, notamment les bandeaux, sont parfois signés - Beugnet, Caron, Cotte, Gritner, Le Sueur, Papillon -, et leurs noms peuvent aider à identifier les origines géographiques d'un ouvrage, au moins si l'on sait où ils habitaient. Les ornements peuvent servir également à distinguer entre deux éditions qui semblent presque identiques; deux éditions de la Péruvienne, portant le nom de Powell de Dublin, sont très similaires, mais les bandeaux de la première page, dans l'une représentant un chien, dans l'autre une corbeille, sont différents (P.17, P.18 et planche 3).

La fin du siècle a témoigné de la quasi-disparition des ornements.

Les estampilles. Une estampille indique que l'édition en question a bénéficié d'une permission simple. En 1777, un édit royal a créé cette permission officielle pour les livres dont le privilège avait expiré. L'estampille porte le nom du lieu, la date et la signature de l'inspecteur de la Librairie, en l'occurrence Havas et Clavel (P.53, P.54, P.56 et planche 4). À la fin du livre on trouve le texte de la permission qui indique le nombre d'exemplaires qu'on a autorisés (P.65 et planche 5). Le regretté Bob Dawson a consacré tout un livre à ce sujet ${ }^{11}$.

Le papier et le filigrane. Le filigrane peut disqualifier une édition comme candidate pour l'édition originale. Parmi les treize éditions de

11. Voir Robert L. Dawson, The French Booktrade and the "permission simple" of 1777; Copyright and Public Domain, with an Edition of the Permit Registers, Oxford, Voltaire Foundation, coll. «SVEC», 301, 1992. 


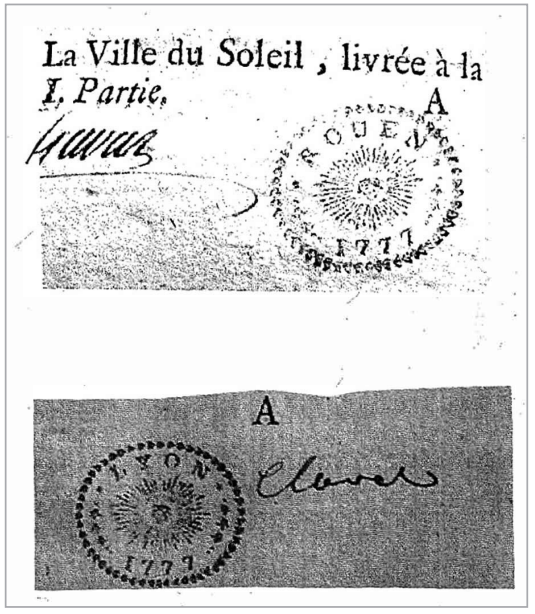

Planche 4

\section{PERMISSION SIMPLE.}

IEAN-JACQUES DE I'IDAUD, Marquis de Velleron, Comee de la Batie \& Monguenins, Seigneur de Fargues, Cairanne, Bivier, la Maifon-forle ile Monstives Es antres Places, Confeiller d'Étens ordinaire E au Confeil privé, Diredeur général de In Librairie, E Dodeur d'honneur de la Faculee de Droit.

$\mathrm{V}$

U l'orticle VII de l'Arrte dn Conseil du 30 Aot I777 portant rtelement pour ta durie des Privileges en Librairie, en sertu des pouroirs à Nous donnés parledit Arret : NoUs permetrons au fieur JEAN RAC.INF, Libraire at Ronen, de faire fjire t:ne édition de l'Ouvrage qui a pintr tirce: Letrotes Féris. yiennes. Lanuetle editinn tera titee th lepe cent cint-

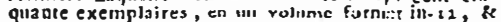
Geráfinie dans le délai de tix mois, a la chatge par ledir fieur de reprefenter a l'Inflyedeur de la Chambre Syndicale de Rouen, la quittance exigée par les articles VIII \& IX du méme Artet; d'stertir Icủit Jnspecteur du jour oú l'on conmencern l'imprestion dudit Ourrage, au defir de l'article X XiI de l'Arrít ủu ConStil du 30 Aoult 1777 , portant fupprefion R créations de dif̈rentes Chambres Syndieales; de fise ladise éditionabfolument conforine à celle d' Anifterdamin-y. d'en remeterc, conforinément à l:'Arrêt du Confeil du 16 Avril $1-83$, ncuf exemplaircs at:x muins les Oniciers de la Clinubre Syndic:ale de loosen, d'imprimer la Préfente permifion a la tin dul Iivre, \& de la faire enregiftrer dans deux mnis, pour tout delai, fur les regiftres de ladite Chatnbre Syndicale de Rowen, le. rout aे peine de nulliré.

DONNE à Paris, le 27 Janvier 1787 .

$\mathrm{V}$ I D A U D.

Par Monteinneur.

$D U M I R \wedge I L$.

Regifle fur le Regifire de la Chambre Syndi-. dicale de Rouen, fol. $39, N^{\circ} .237$, conformément anx Reiglements do la I.itrairic. A Ruv:erns. o premier Firrice egs

LOUIS OURSEL, Syndis,

Planche 5

la Péruvienne portant l'adresse A PEINE, deux portent le filigrane de Tacusel d'Orange (P.7 et P.8), indiquant probablement une origine avignonnaise ; elles ne peuvent donc pas être l'originale, qui fut imprimée à Paris.

On trouve des éditions de luxe de la Péruvienne imprimée sur du papier vélin. Le tirage de ces éditions, dont le papier coûtait cher, était généralement assez limité et le prix relativement élevé. L'émission Bleuet-Didot de 1797 (P.79) sur papier vélin coûtait 12 livres, alors que la même chose sur papier ordinaire ne coûtait que 6 livres.

Le filigrane indique parfois le lieu de sa fabrication. Par exemple, un papier avec le filigrane de DUVAL, GÉNÉRALITÉ DE ROUEN est nécessairement rouennais, même si l'on trouve sur la page de titre l'adresse d'Amsterdam (voir P.25, P.27, P.30, P.38, P.41 et P.43). 
L'approbation et le privilège. Un règlement de la Librairie exigeait la publication de l'approbation et du privilège d'un ouvrage autorisé. L'édition originale de Cénie (C.1A) et l'édition révisée de la Péruvienne (P.22A) en ont, mais l'édition originale de la Péruvienne n'en a pas en raison de l'interdiction des romans ${ }^{12}$. Il va sans dire que les pirates reproduisaient approbation et privilège, auxquels ils n'avaient aucun droit (P.46).

Errata. Tout éditeur moderne doit tenir compte de l'errata des premières éditions. L'édition révisée de la Péruvienne de 1752 (P.22A) en comporte un (planche 6), mais il ne fut ajouté quavec un délai de deux semaines, de sorte qu'il ne figure pas dans les exemplaires déjà vendus. L'édition Garnier-Flammarion se dit "définitive ${ }^{13}$ ", mais ne l'est pas, puisque ses éditeurs n'ont pas vu l'errata, que je n'ai trouvé que dans les exemplaires de Lunéville, de Toronto et de Yale. Heureusement, Jonathan Mallinson l'utilise dans son édition critique ${ }^{14}$, suivie de celle de la M.L.A. ${ }^{15}$.

Les éditions révisées. Seule la Péruvienne a été révisée, d'abord en 1752 (P.22A), puis en 1760 (P.29A). Mme de Graffigny déplorait la fâcheuse tendance des pirates à republier son roman avec deux suites apocryphes. En y ajoutant trois nouvelles lettres, elle espérait non seulement revendre son roman à un prix avantageux, mais encore montrer qu'il n'avait pas besoin des suites. C'est d'ailleurs l'un des buts d'une nouvelle Introduction historique, érudite et anonyme, qui est en fait de son ami Antoine Bret. Le faux-titre et le titre deviennent donc plus explicatifs: «Augmentée de plusieurs lettres et d'une Introduction à l'histoire ». La préface est également révisée, mais ne contient aucune réponse aux critiques de l'édition originale.

Duchesne a eu du mal à écouler son stock, car de nouvelles émissions, identiques sauf pour le changement de date sur la page de titre gravée, sont publiées en 1753, 1754, 1755 et 1756. On aurait donc tort de

12. Voir Françoise Weil, L'interdiction du roman et la librairie 1728-1750, Paris, Aux amateurs des livres, 1986.

13. Guilleragues, Lettres portugaises, et Graffigny, Lettres d'une Péruvienne, éd. Bernard Bray et Isabelle Landy-Houillon, Paris, Garnier-Flammarion, 1983, p. 242 et 244 .

14. Françoise de Graffigny, Lettres d'une Péruvienne, éd. Jonathan Mallinson, Oxford, Voltaire Foundation, coll. «Vif», 2002.

15. Françoise de Graffigny, Lettres d'une Péruvienne, $4^{\mathrm{e}}$ impression, avec corrections, éd. Joan DeJean et Nancy K. Miller, New York, M.L.A., 2002 [1993]. 


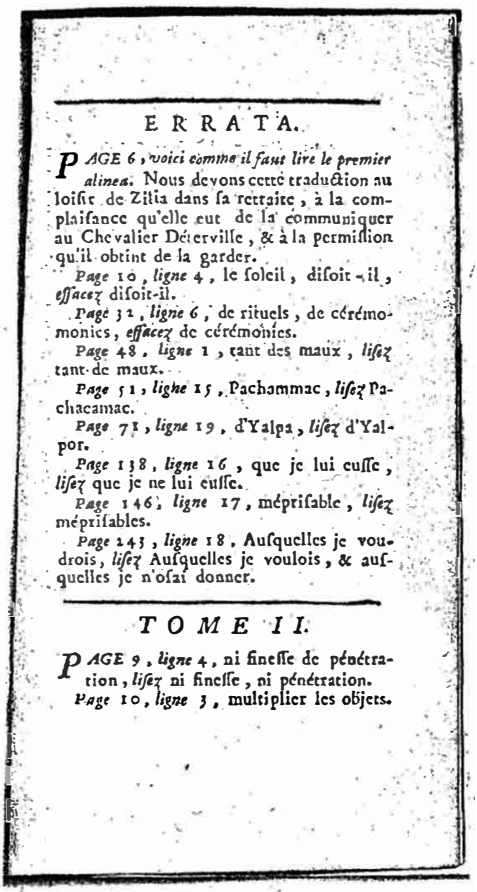

Planche 6

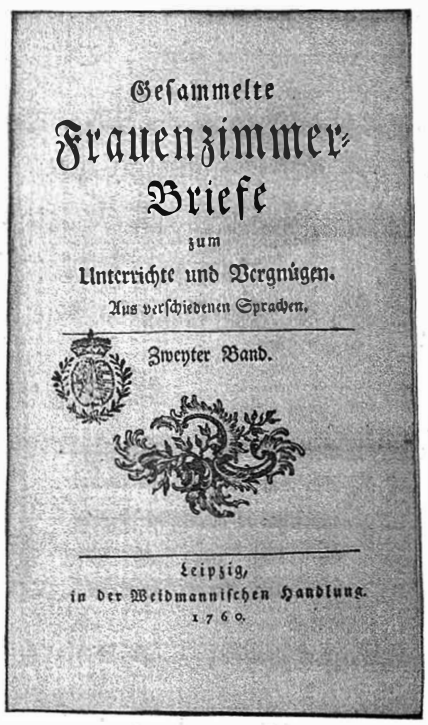

Planche 7

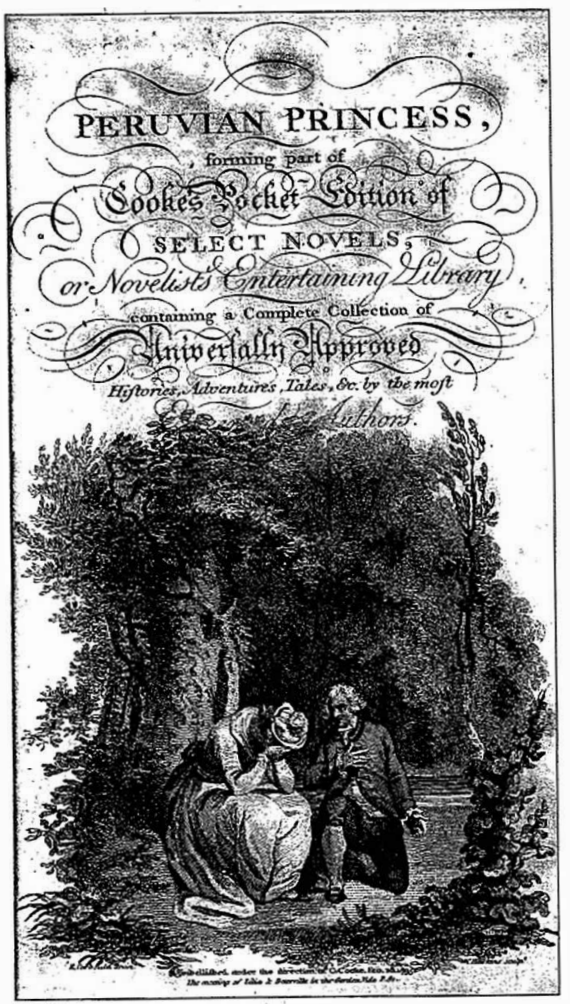

Planche 8 
croire que ces cinq émissions indiquent la grande popularité de cette édition; c'est plutôt le contraire.

La nouvelle édition de 1760 comporte une Vie anonyme de Mme de Graffigny, dont les auteurs sont multiples - Devaux, Duclos, La Touche, etc. Comme je l'ai dit, on y corrige le plus grand nombre des erreurs figurant dans l'errata de 1752 .

Les traductions. Avant 1855, on a traduit la Péruvienne en neuf langues (allemand (planche 7), anglais (planche 8), danois, espagnol, italien, polonais, portugais, russe et suédois), Cénie en six langues (allemand, anglais, danois, espagnol, italien et néerlandais), et La fille d'Aristide uniquement en allemand. Les traductions sont rarement anonymes et généralement assez fidèles, mais il y a des exceptions. Une traduction anglaise de Miss R. Roberts (P.49) ajoute 29 nouvelles lettres de son cru, convertit Zilia au christianisme, et la marie à Déterville. La première traduction espagnole (P.73) ajoute une nouvelle lettre dans laquelle Zilia décrit sa conversion au catholicisme et justifie la conquête cruelle du Pérou par les Espagnols en termes du salut de ses habitants. Les traductions italiennes comportent souvent des accents prosodiques pour faciliter la prononciation. On publiait aussi des éditions bilingues - français-anglais une fois (P.89.1), mais surtout français-italien, dont le grand nombre laisse supposer qu'on les employait pour enseigner l'italien.

Cet article n'a ni thèse, ni fil conducteur, ni conclusion. Dans ce survol de certains aspects bibliographiques de l'œuvre de Mme de Graffigny, j’espère pourtant avoir démontré à quel point le paratexte bibliographique peut éclairer les circonstances de la composition, de la publication et de la réception des différentes éditions de ses ouvrages et jeter une nouvelle lumière sur les textes mêmes. Enfin, bibliographes, collectionneurs, bibliothécaires et lecteurs pourront utiliser cet article pour mieux évaluer les éditions qu'ils possèdent ou qu'ils envisagent de se procurer.

\section{Liste des éditions citées}

\section{Lettres d'une Péruvienne}

P.1. A Peine [Paris, Veuve Pissot, décembre 1747 ou janvier 1748]. 12 $2^{\circ} . \pi 1 \mathrm{a}^{4}$ $\mathrm{A}-2 \mathrm{E}^{8.4}{ }_{2} \mathrm{~F}_{1} ;[2]$ [i] ij-viij [1] 2-337 [1] p.

P.5. A Peine [Lyon, Delaroche, 1748]. 12 . . $a^{6}$ A-2E $E^{8.4}$; [i-iv] v-xij [1] 2-336 p. 
P.7. A Peine [1748]. $12^{\circ} \cdot a^{6} \mathrm{~B}-2 \mathrm{~A}^{6} 2 \mathrm{~B}^{4}$; [i-iv] v-x 1-285 [1] p.

P.8. A Peine [1748]. $12^{\circ} \cdot a^{8} \mathrm{~B}^{4} \mathrm{C}-2 \mathrm{~A}^{8.4}{ }_{2} \mathrm{~B}^{4}$; [i-iv] v-x 1-285 [1] p.

P.9. A Peine [Liège, Kints, 1748]. 12 . $\mathrm{a}^{4} \mathrm{~A}-\mathrm{Y}^{8.4} \mathrm{Z}^{8}$ (-Z8) ; [2], [i] ij-vj [1] 2-278 p.

P.17. Letters written by a Peruvian princess, Dublin, S. Powell for Thomas Moore, 1748 .

P.18. Letters written by a Peruvian Princess, Dublin, S. Powell for Thomas Moore, 1748.

P.22A. Lettres d'une Péruvienne, avec Cénie, Paris, Duchesne, 1752, 2 vol.

P.25. Lettres d'une Péruvienne, avec la première Suite et les Lettres d'Aza, Amsterdam, Aux dépens du délaissé [= Rouen], 1755, 2 vol.

P.27. Lettres d'une Péruvienne, avec la première Suite et les Lettres d'Aza, Amsterdam, Aux dépens du délaissé, 1758, 2 vol.

P.29A. Lettres d'une Péruvienne, avec les Lettres d'Aza, Paris, Duchesne, 176o, $2 \mathrm{vol}$

P.30. Lettres d'une Péruvienne, avec la première Suite et les Lettres d'Aza, Amsterdam, Aux dépens du délaissé [= Rouen], 1760, 2 vol.

P.33. Briefe einer Peruanerin, avec la première Suite et les Briefe des Aza, dans Gesammelte Frauenzimmerbriefe zum Unterrichte und Vergnügen, Leipzig, Weidmann, 1760, vol. 2.

P.38. Lettres d'une Péruvienne, avec la première Suite et les Lettres d'Aza, Amsterdam, Aux dépens du délaissé [= Rouen], 1764, 2 vol.

P.41. Lettres d'une Péruvienne, avec la première Suite et les Lettres d'Aza, Amsterdam, Aux dépens du délaissé [= Rouen], 1767, 2 vol.

P.43. Lettres d'une Péruvienne, avec la première Suite et les Lettres d'Aza, Amsterdam, Aux dépens du délaissé [= Rouen], 1770, 2 vol.

P.46. Lettres d'une Péruvienne, avec les Lettres d'Aza, Paris, Veuve Duchesne [= Rouen], 1773 .

P.49. The Peruvian letters, avec la première Suite et une suite due à R. Roberts, London, T. Cadell, 1774, 2 vol.

P.53. Lettres d'une Péruvienne, avec la première Suite et les Lettres d'Aza, Amsterdam, Aux dépens du délaissé [= Rouen], 1775, 2 vol.

P.54. Lettres d'une Péruvienne, avec la première Suite et les Lettres d'Aza, Amsterdam, Aux dépens du délaissé [= Rouen], 1775, 2 vol.

P.55. Genève [= Lyon], 1777.

P.56. Péruvienne / Peruviana, Parigi, Duchesne [= Lyon], 1777.

P.65. Lettres d'une Péruvienne, avec la première Suite et les Lettres d'Aza, Rouen, Jean Racine, 1787, 2 vol.

P.73. Cartas de una Peruana, Valladolid, Viuda de Santander, 1792. 
P.79. Paris, Joseph Bleuet et Pierre Didot l'aîné, An V. 1797 [= 1798], 2 vol. P.8o. Letters of a Peruvian princess, avec la première Suite et les Letters of Aza, London, C. Cooke, [1798].

P.89.1. Lettres d'une Péruvienne / Letters of a Peruvian princess, avec la première Suite et les Lettres d'Aza / Letters of Aza, Paris, Durand, 18o2, 2 vol.

\section{Cénie}

C.1A. Paris, Cailleau, 1751. 12. iv, 133, [11] p.

C.8. Cenia, London, Reeve, 1752.

C.9. Eugenia, London, Millar, 1752.

C.18. Cenie, blyspel, Amsterdam, Duim, 1760.

\section{La fille d'Aristide}

A.3. Vienne, Ghelen, 1759. 